\title{
EchoGéo
}

52 | 2020

Ho Chi Minh Ville, terrain de jeu(x) métropolitain(s)

\section{Ho Chi Minh Ville, des inondations à la submersion...}

Georges Vachaud, Nicolas Gratiot et Tien Dung Tran Ngoc

\section{OpenEdition}

Journals

Édition électronique

URL : https://journals.openedition.org/echogeo/19473

DOI : $10.4000 /$ echogeo. 19473

ISSN : 1963-1197

Éditeur

Pôle de recherche pour l'organisation et la diffusion de l'information géographique (CNRS UMR 8586)

Référence électronique

Georges Vachaud, Nicolas Gratiot et Tien Dung Tran Ngoc, « Ho Chi Minh Ville, des inondations à la submersion... », EchoGéo [En ligne], 52 | 2020, mis en ligne le 15 juillet 2020, consulté le 10 août 2021. URL : http://journals.openedition.org/echogeo/19473; DOI : https://doi.org/10.4000/echogeo.19473

Ce document a été généré automatiquement le 10 août 2021.

EchoGéo est mis à disposition selon les termes de la licence Creative Commons Attribution - Pas d'Utilisation Commerciale - Pas de Modification 4.0 International (CC BY-NC-ND) 


\title{
Ho Chi Minh Ville, des inondations à la submersion...
}

\author{
Georges Vachaud, Nicolas Gratiot et Tien Dung Tran Ngoc
}

\section{Introduction}

1 Comme la plupart des mégacités d'Asie du Sud Est, Ho Chi Minh Ville (HCMV) est, avec une altitude moyenne inférieure à $1,5 \mathrm{~m}$ au dessus du niveau moyen de la mer, située dans une zone côtière de très faible altitude. Cette zone, fréquemment nommée « Low Elevation Coastal Zone (LECZ) » par les démographes et les économistes est au cœur de nombreux enjeux de développement urbain, et est particulièrement exposée aux risques globaux.

2 D'une part cette mégacité connait un climat contrasté, marqué par une forte saisonnalité et des pluies particulièrement intenses durant l'été, d'autre part elle est vulnérable à l'immersion des eaux côtières, qui se produit lors de chaque marée haute et s'accentue progressivement sous l'effet couplé de la subsidence des sols et de l'élévation du niveau des mers.

3 La très forte croissance économique et démographique de la ville, commencée au XIX siècle, augmente de façon exponentielle depuis 1986, date de transition à l'économie de marché. Son rythme annuel avoisine en 2019 6,8\% du PIB. Toutefois cette croissance est fragilisée par une exposition progressive au risque d'inondation, due à l'étalement urbain et à l'artificialisation des sols qui réduit la résilience naturelle du milieu.

\section{La situation actuelle}

Le $\mathrm{XXI}^{e}$ siècle est celui des transitions, accompagnées ou contraintes, avec un paradoxe clé à gérer: celui d'une concentration galopante des populations dans les zones urbaines localisées dans les espaces littoraux qui sont les plus vulnérables aux changements globaux et au mode d'occupation des terres. Ce paradoxe concerne bon 
nombre de pays, mais est exacerbé en Asie car ce continent concentre simultanément trois quarts de la population côtière et trois quarts de la population urbaine avec un constat clair: parmi les dix villes les plus densément peuplées au monde, huit sont situées en Asie ${ }^{1}$. Ces mégacités sont confrontées à des risques environnementaux grandissants, avec des répercussions à craindre sur l'économie régionale et mondiale.

5 Avec environ 10 millions d'habitants, HCMV a vu sa taille et sa population augmenter considérablement au cours des deux dernières décennies (Kontgis et al., 2014). La ville s'étend aujourd'hui sur un continuum urbain de plus de $800 \mathrm{~km}^{2}$ et sa population croît actuellement à un rythme de 3,2\% par an. Au-delà de ses limites administratives, la zone urbaine s'étend également dans les provinces voisines, sur un continuum urbain de plusieurs dizaines de kilomètres englobant ainsi une population totale d'environ 20 millions d'habitants (Chatel et Moriconi-Ebrard, 2018). En moyenne, $16 \mathrm{~km}^{2}$ sont urbanisés chaque année depuis l'an 2000 (Ministère des Ressources naturelles et de l'Environnement, 2013).

Cette urbanisation étendue et rapide a eu des effets majeurs sur le cycle hydrologique : à ce jour $90 \%$ de l'espace urbain est imperméable, (Tran Ngoc, 2016). En outre, des taux d'affaissement du niveau du sol (subsidence) importants (jusqu'à $3 \mathrm{~cm} / \mathrm{an}$, illustration 1) sont observés en raison de la surexploitation des eaux souterraines et de la construction intensive sur des alternances de couches géologiques de limon et d'argile non consolidées, alors que $65 \%$ de la surface urbaine se situait à une altitude inférieure à 1,5 m au-dessus du niveau de la mer en 2009 (VCAPS, 2013).

Illustration 1 - Vitesse de subsidence dans la métropole

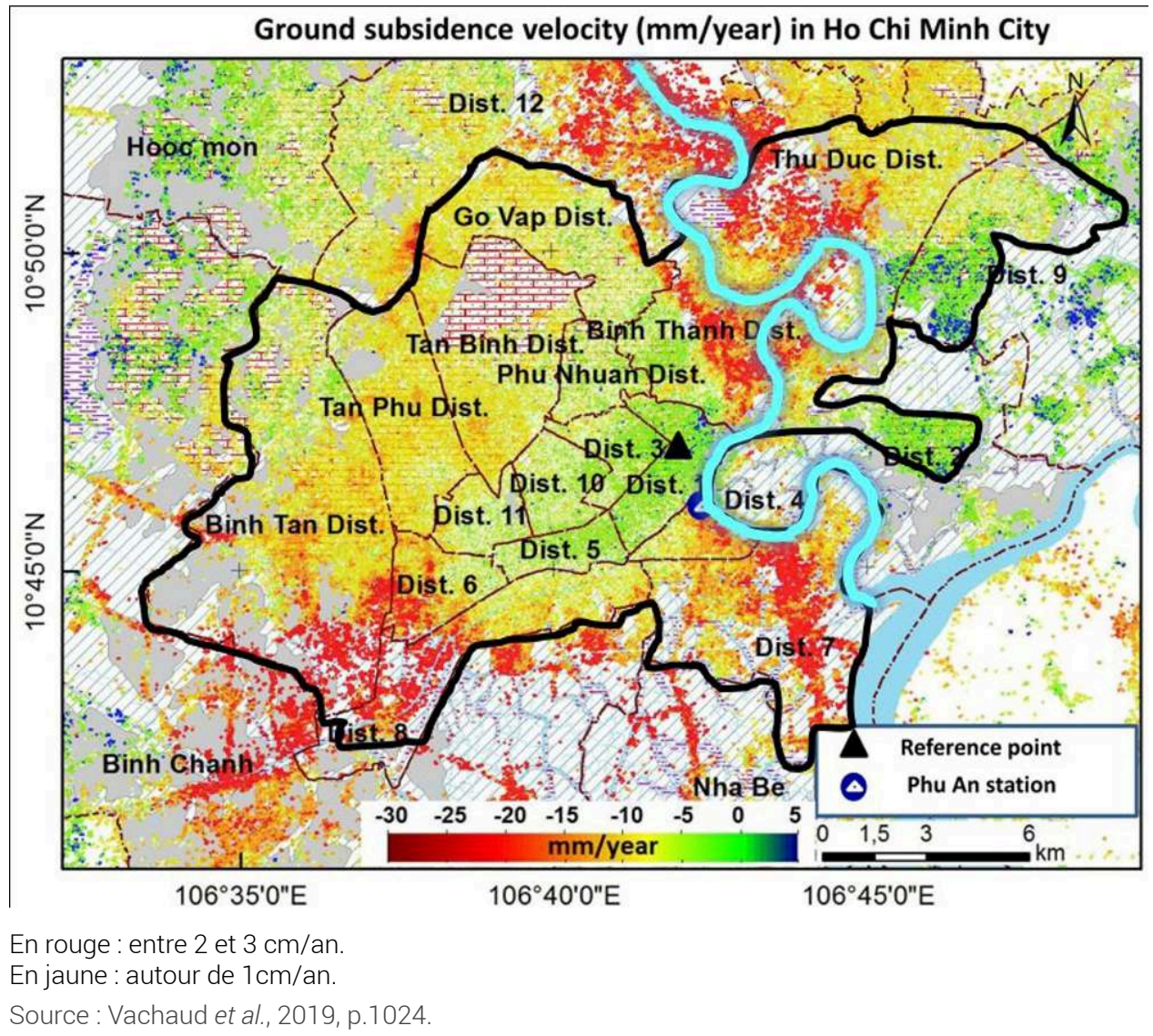

EchoGéo, 52 | 2020 
7 La mégacité est de ce fait soumise de façon récurrente à de graves inondations d'août à septembre, avec un impact très important sur le trafic urbain (notamment du fait d'embouteillages monstres de scooters et motos bloqués par les eaux), la pollution atmosphérique et certains dommages aux habitations et aux sites industriels. Elles résultent de la conjonction de trois conditions hydrométéorologiques simultanées : fortes pluies locales, débit élevé des rivières Saigon et Dongnai et marées hautes. Elles sont aggravées par l'inefficacité des infrastructures d'assainissement. Occasionnellement, comme dans le cas du typhon Usagi (18-25 novembre 2018), des inondations exceptionnelles peuvent survenir en octobre-novembre.

\section{Tendances et impacts}

Sur la base des mesures et observations effectuées au cours de la dernière décennie, il est possible d'établir des tendances d'évolution sur l'occurrence et la fréquence des zones inondées en fonction des précipitations, des marées, dont l'influence se fait ressentir bien au delà de la métropole, et de l'urbanisation.

La pluviométrie régionale est en moyenne annuelle est de $1600 \mathrm{~mm}$, avec une fluctuation interannuelle d'environ $350 \mathrm{~mm}$ sans qu'une tendance de variation significative soit détectable (Dao et Hoang, 2016). Toutefois, à l'échelle journalière et locale, une nette augmentation interannuelle a été observée pour les précipitations quotidiennes supérieures au seuil de $50 \mathrm{~mm} /$ jour. Cette augmentation apparaît clairement dans l'illustration 2 (Ho et al., 2014) et se traduit par une modification des statistiques : l'occurrence d'un événement de $100 \mathrm{~mm}$ est passée d'une fois tous les cinq ans à une fois par an au cours de cette période.

Illustration 2 - Variation interannuelle du pic de pluie journalière

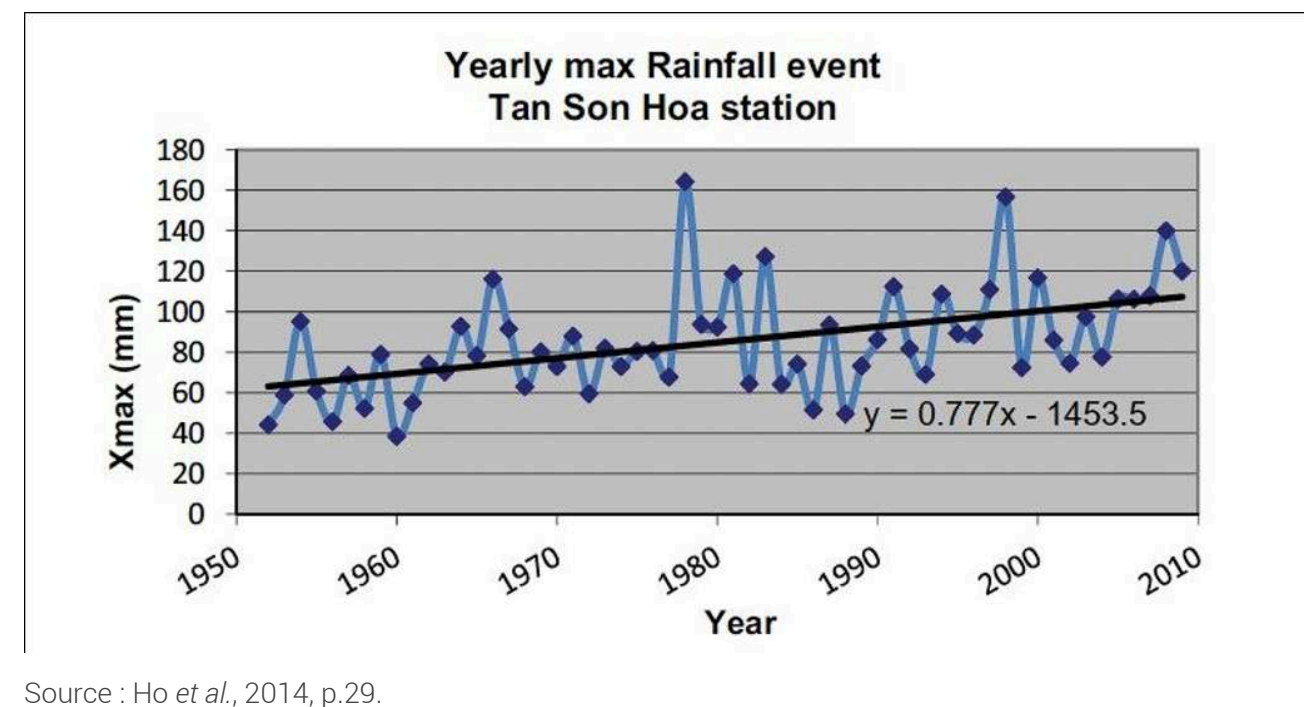

Les marées de leur côté ont un marnage journalier entre 2,5 et $3 \mathrm{~m}$ dans le centre ville (VCAPS, 2013), sans qu'un changement spécifique dans leur amplitude puisse être détecté, l'élévation du niveau de la mer observée sur la période 1993-2003 pour la ligne côtière du Sud Vietnam étant seulement de 0,031+/- 0,007 m (Pham et Furukawa, 2007), soit environ $3 \mathrm{~mm} / \mathrm{an}$. 
Les observations satellitaires régulières (Koudogbo et al., 2012) permettent d'identifier la coexistence de deux typologies d'inondations soit d'origine marine, soit par ruissèlement pluvial (illustrations $3 a$ et $3 b$ ) : les districts urbains de faible altitude proches de la rivière Saigon (en particulier les districts nouvellement urbanisés construits sur des marécages ou des plans d'eau antérieurs) sont essentiellement soumis à un risque d'inondation lié à l'occurrence du cumul de fortes marées avec les fortes pluies (surtout en septembre). Le reste de la ville est plutôt touché par les inondations dues au ruissellement provenant de l'imperméabilisation des sols urbains (Vachaud et al., 2018). Il faut toutefois noter qu'en raison de l'hétérogénéité spatiale de la pluviométrie la distribution des zones inondées est très localisée (Tran Ngoc et al., 2016). En outre, l'inefficacité du système de drainage (pluvial et égouts) ainsi que la subsidence qui réduit d'autant la pente naturelle d'écoulement sont deux problèmes majeurs.

\section{Illustration 3a - Typologie des zones d'inondation par la marée et ou les précipitations}

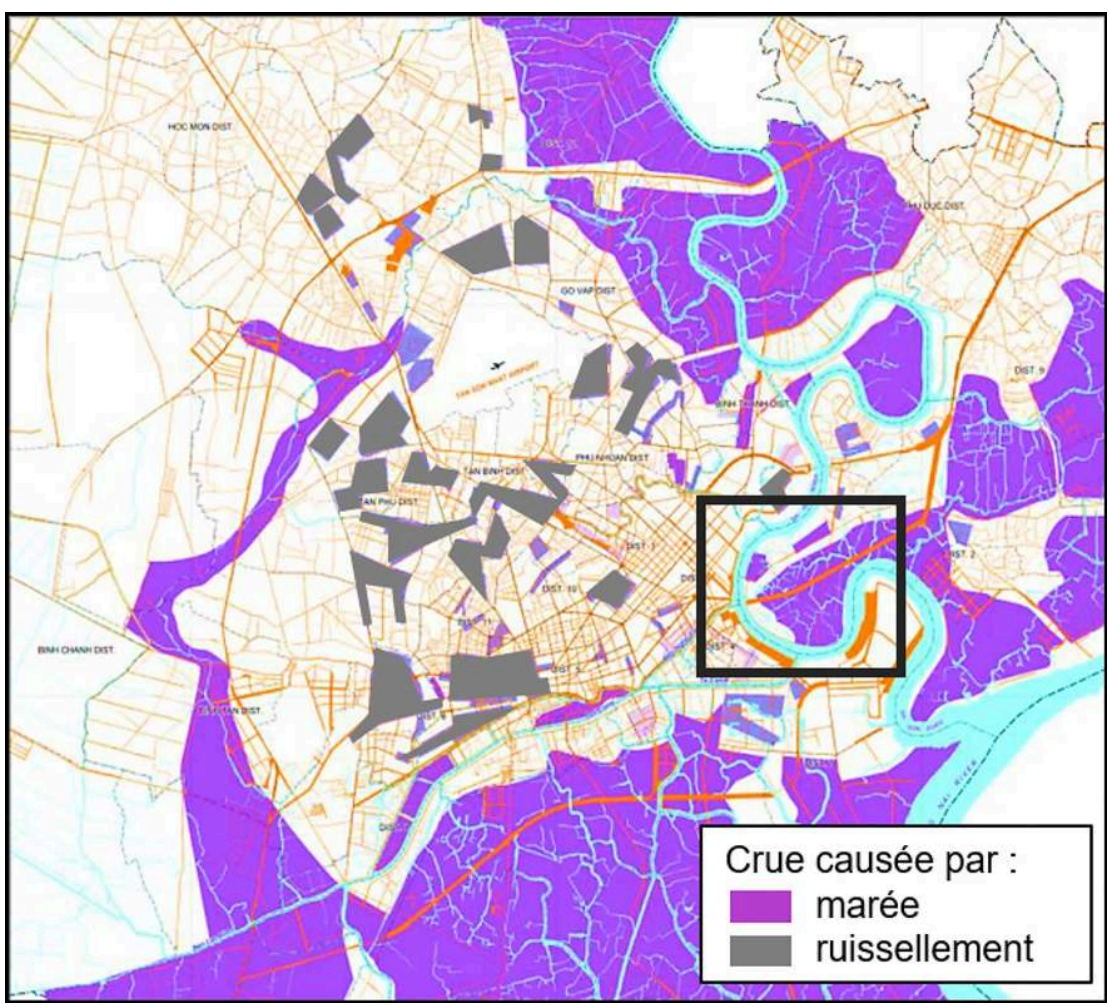

La zone entourée d'un carré correspond à l'illustration 3-b.

Source : Vachaud et al. 2019, p.1024. 
Illustration $3 \mathrm{~b}$ - Vue aérienne du quartier de Thu Thiem et du centre historique ( $1^{\mathrm{er}}$ arrondissement)

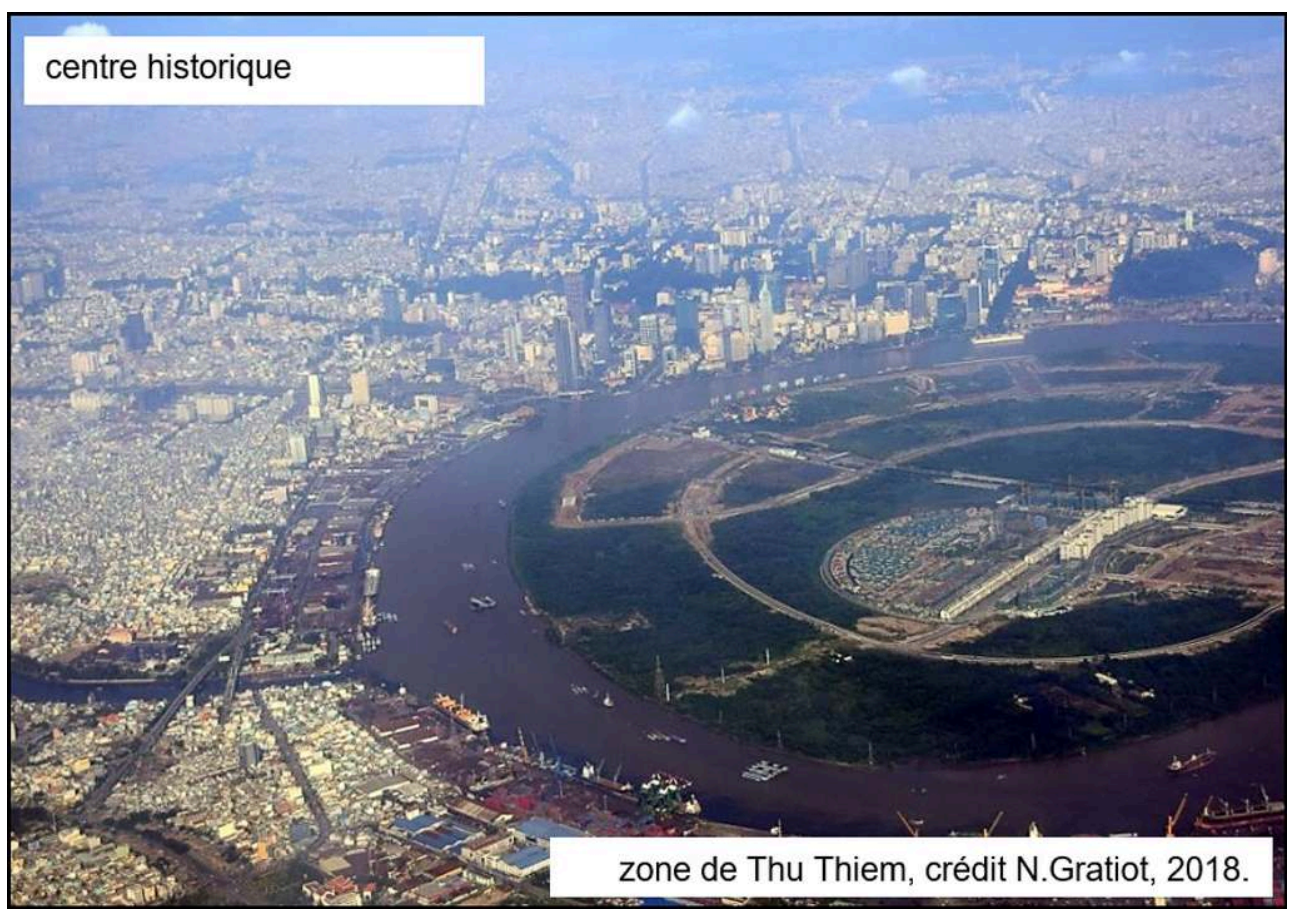

Le quartier de Thu Thiem est promis à un développement urbain écologique et le centre historique très fortement urbanisé.

Auteur : Nicolas Gratiot, 2018

Il est en effet clair que vis-à-vis ces deux aléas le risque essentiel d'inondation est lié à la capacité limitée du système de drainage d'évacuer les eaux. Ce système repose sur deux composantes: d'une part un réseau de canaux (près de $4745 \mathrm{~km}$ ) dont la construction remonte à la fin du XVIII ${ }^{\mathrm{e}}$ siècle, d'autre part un réseau d'égouts (près de $3100 \mathrm{~km}$ ) pour la collecte des eaux pluviales et usées dont la construction a débuté à la fin du XIX ${ }^{e}$ siècle. Une partie des canaux, initialement conçus pour la navigation, ont été couverts pour devenir de grandes avenues (dans les districts centraux); pour le reste des constructions informelles et des dépôts incontrôlés de déchets solides les ont rendus pour l'essentiel inopérants. Par ailleurs, une grande partie des canalisations d'évacuation sont sous-dimensionnées et souffrent d'un très mauvais entretien. Des travaux importants ont déjà été réalisés pour améliorer et remédier à cette situation. Ainsi, entre 2001 et 2015, 1,3 milliard USD ont été investis et le réseau a été étendu de $516 \mathrm{~km} ; 5$ milliards de dollars US supplémentaires ont été prévus pour 2016-2020 (Tran Ngoc, 2016). Une diminution très nette et importante des zones inondées a de ce fait pu être observée à partir de 2007, comme cela est illustré par l'illustration 4, mais depuis 2013 la fréquence des zones inondées augmente de nouveau car la mise en place des réseaux ne peut pas suivre le rythme du développement urbain dans les nouvelles zones périphériques. 


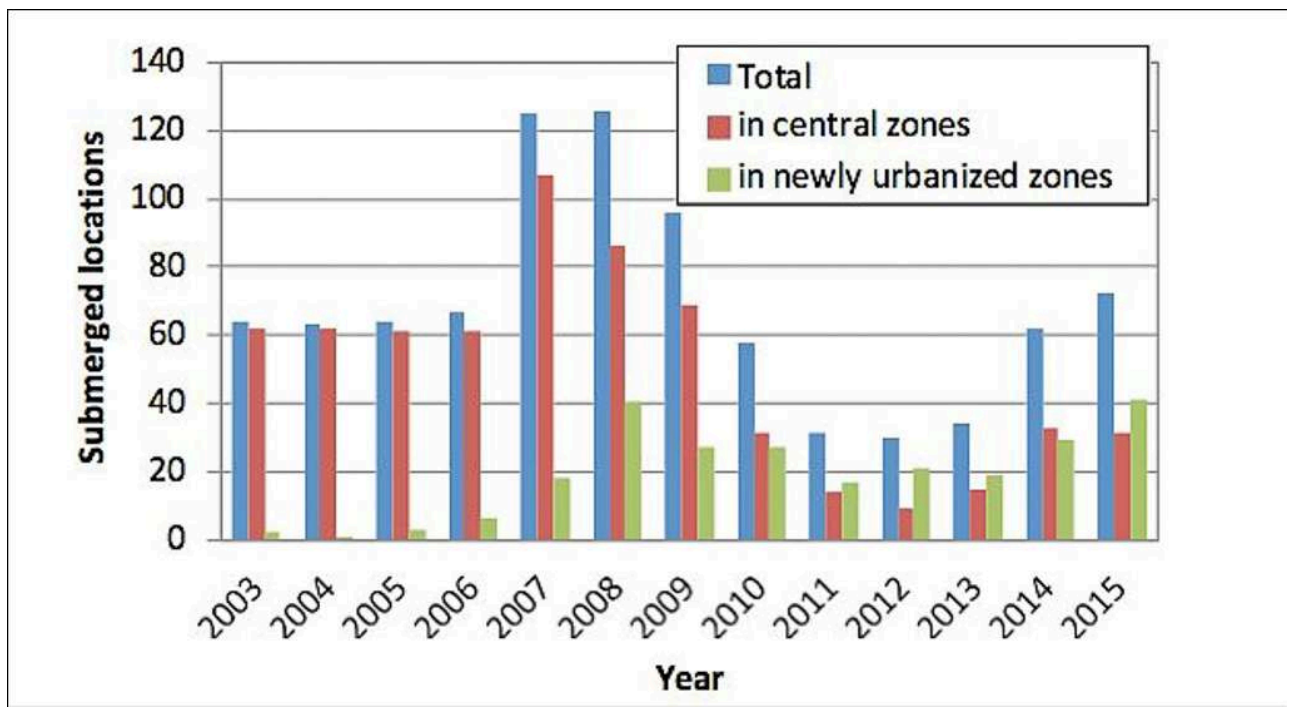

Source : Tran Ngoc et al. 2016.

En outre la forte croissance démographique entraine une très forte demande en eau, satisfaite en grande partie par l'augmentation des pompages. Le nombre de forages (inventoriés) en exploitation est ainsi passé de 100000 environ en 2004 à 200000 en 2016, avec une capacité d'extraction de 1 million de $\mathrm{m}^{3} /$ jour (Nguyen, 2016). Dans plusieurs districts du Sud de la métropole le rabattement de la nappe phréatique a été de l'ordre de $20 \mathrm{~m}$ s'accompagnant d'une subsidence voisine ou supérieure à $4 \mathrm{~cm} / \mathrm{an}$ (Minderhoud, 2017), soit un ordre de grandeur supérieur à la remontée océanique. De ce fait la pente naturelle d'évacuation des eaux drainées diminue régulièrement.

\section{Évolution prévisible}

Deux points essentiels posent question concernant l'évolution possible de la situation de la ville face aux risques hydrologiques: quel impact du changement climatique serait attendu? quelles pourraient être les conséquences de la poursuite du développement urbain?

\section{Ho Chi Minh Ville face au changement climatique}

Différents scénarii d'émission de gaz de serre ont été utilisés par le groupe de travail du Groupe d'experts intergouvernemental sur l'évolution du climat, le GIEC ( $5^{\text {eme }}$ Rapport Changements climatiques et leur évolution future, publication IPCC 2015), pour faire des projections selon la taille de la population, l'activité économique, les modes de vie, la consommation énergétique, l'utilisation des terres, la technologie et la politique climatique. Les deux scénarii les plus utilisés pour simuler l'évolution de la pluviométrie et de la remontée du niveau océanique sont :

- RCP 4.5 (Representative concentration pathway) correspondant à une modification du bilan radiatif de la planète de 4,5 watts $/ \mathrm{m}^{2}$ du fait de l'évolution de la concentration en gaz de serre et visant à contraindre les émissions pour maintenir le réchauffement planétaire à la surface du globe à la fin du XXI siècle dans une plage voisine de $2,6^{\circ} \mathrm{C}$ au delà des températures préindustrielles grâce au développement de technologies 
propres,

- RCP 8.5 correspondant une modification du bilan radiatif de 8,5 watts $/ \mathrm{m}^{2}$ et résultant de l'inaction par rapport aux conditions d'émission actuelles, Business as usual, ce qui conduirait à une augmentation moyenne de température voisine de $4,8^{\circ} \mathrm{C}$.

Concernant l'impact du changement climatique sur les précipitations, on trouvera sur l'illustration 5 les résultats obtenus par l'Environmental Change Institute Oxford University, avec, dans une première phase (1960-2010), une comparaison avec les données mensuelles disponibles et, dans une seconde phase, une projection jusqu'en 2100 (Mc Sweeney et al., 2009). Il n'y a pas de différences notables entre les scénarios, ni de changement attendu des tendances. On notera qu'à l'échelle mondiale ce résultat est général à la zone intertropicale. On peut noter que pour le scénario extrême (RCP 8.5) l'augmentation moyenne annuelle des précipitations ne seraient en 2100 que de $0,7 \%$ par rapport à l'actuel.

Illustration 5 - Prédiction des pluies mensuelles au Sud Vietnam en fonction des scénarios climatiques

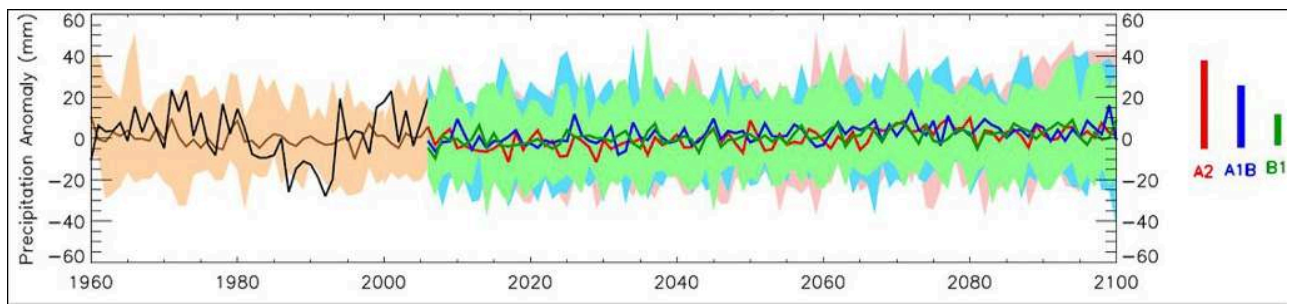

A gauche, en jaune : mesures

A droite, en vert : tendances modélisées.

Source : Mc Sweeney et al., 2009, consulté en ligne.

17 Ce résultat peut sembler contradictoire aux observations reportées dans l'illustration 2 , mais celles ci ne concernent toutefois que l'augmentation des précipitations extrêmes à l'échelle journalière, qui sont le principal moteur de la dynamique des crues. Dans l'état actuel il apparaît que d'autre processus devraient être pris en compte dans ces modèles pour la génération des pluies extrêmes: probablement les oscillations australes d'El Niño (ENSO- El Niño Southern Oscillation) et surtout l'impact de l'urbanisation sur la création d'ilots de chaleur locaux, point sur lequel nous reviendrons.

L'autre impact mis en avant concerne l'élévation du niveau de la mer (SLR, Sea Level Rise) actuellement présenté comme un des problèmes majeurs pour le delta du Mékong et la zone côtière de très faible altitude. Le SLR est très fortement lié à la température pour deux raisons essentielles: un tiers de la hausse observée depuis 1990 est due à l'expansion thermique des océans du fait de l'augmentation globale de lié à la température marine, deux tiers (jusqu'à présent) sont dus à la fonte des glaces polaires. Dans tous les cas, le taux d'élévation du niveau océanique dans la zone côtière du Sud Vietnam devrait passer d'environ 1,6 mm/an, mesurés actuellement, à environ 6 à $10 \mathrm{~mm} / \mathrm{an}$, selon les scénarios climatiques, avec pour la fin du siècle une augmentation de niveau allant de 0,3 à $1 \mathrm{~m}$, et une grosse incertitude concernant la fonte des glaces polaires (Jevrejeva et al., 2016) 


\section{Développement urbain}

19 Ho Chi Minh Ville est soumise à des contraintes géo-climatiques particulières et à une dynamique de croissance impliquant à la fois la densification urbaine et l'expansion péri-urbaine au détriment des zones rurales et des zones humides naturelles. Cette expansion, amplifiée par l'afflux croissant de "migrants environnementaux" (Chapman, 2019), (essentiellement des agriculteurs chassés du Delta du Mékong par la salinisation des sols et venant s'installer au rythme d'environ 28000 par an dans de nouvelles zones), se fait conjointement à un assèchement des rizières et des marais, sans que soit pris en compte au préalable leur utilité dans l'atténuation des risques de submersion/inondation par les eaux de ruissellement localisées et la crue de rivières Saigon et Dong Nai

Cette évolution démographique peut avoir deux autres conséquences importantes sur les risques d'inondation et de submersion. D'une part la densification s'accompagne d'un très fort accroissement du trafic et de la construction de très nombreux immeubles de bureau et d'habitation. L'émission des gaz d'échappement, l'absorption du rayonnement solaire par les bâtiments et la chaleur émise par la climatisation, produisent des changements importants dans la micro météorologie locale (ilot de chaleur urbain, Crutzen, 2004) qui peuvent expliquer l'augmentation des précipitations convectives de haute intensité mesurée depuis les années 2000. D'autre part le développement urbain s'accompagne de la construction d'un très grand nombre d'immeubles (gratte-ciels) et du développement d'un réseau de lignes de métro souterraines. Ces travaux s'accompagnent nécessairement de pompages de haut débit dont les conséquences sur la subsidence s'ajoutent à celles déjà notées précédemment pour les forages domestiques. De plus le poids des bâtiments conduit à un tassement mécanique des sols qui amplifie cette tendance. qui ne font qu'accroître ce processus. Le mécanisme de consolidation et de tassement des sols étant irréversible dans l'état actuel d'occupation du milieu la subsidence du sol ne semble pas pouvoir être bloquée.

C'est donc probablement davantage le développement urbain qui serait à l'origine de l'accroissement des risques d'inondation, cela du fait des effets cumulés de l'augmentation des épisodes pluvieux intenses résultant de la formation d'ilots de chaleur, de la diminution des surfaces susceptibles d'absorber les eaux de surface et de l'affaissement des sols conduisant à l'inefficacité des systèmes d'évacuation des eaux.

Dans un proche avenir, la situation pourrait devenir particulièrement délicate avec le risque d'une submersion permanente de plusieurs districts de la métropole en fin d'étédébut d'automne. Au rythme actuel, et dans environ 50 ans, les districts se trouvant dans les zones les plus basses pourraient être submergés par 0.3 à $0.5 \mathrm{~m}$ d'eau marine $\mathrm{du}$ fait du couplage entre perte d'environ $1 \mathrm{~m}$ d'altitude et remontée d'environ 0,3-0,5 $\mathrm{m}$ du niveau océanique due au changement climatique, processus dénommé "variation effective du niveau océanique (ESLR, Effective Sea Level Rise, Minderhoud, 2017)! Ho Chi Minh Ville se trouverait dès lors dans une situation aussi critique que Djakarta où plusieurs quartiers côtiers en dessous du niveau marin sont sous la protection aléatoire de digues (Abidin et al., 2015).

23 Cette situation n'est pas localisée et pourrait concerner toute la zone incluant le delta du Mékong et la métropole si l'on se réfère à l'article très récent de Minderhoud et al. (2019) (illustration 6) avec comme conséquence la migration vers l'intérieur de plus de 
10 millions de personnes et la perte de productions rizicoles et horticoles représentant actuellement près de $60 \%$ de la production nationale.

Illustration 6 - Impact d'une variation relative du niveau de mer de $1 \mathrm{~m}$ sur la distribution des zones submergées du Delta du Mékong

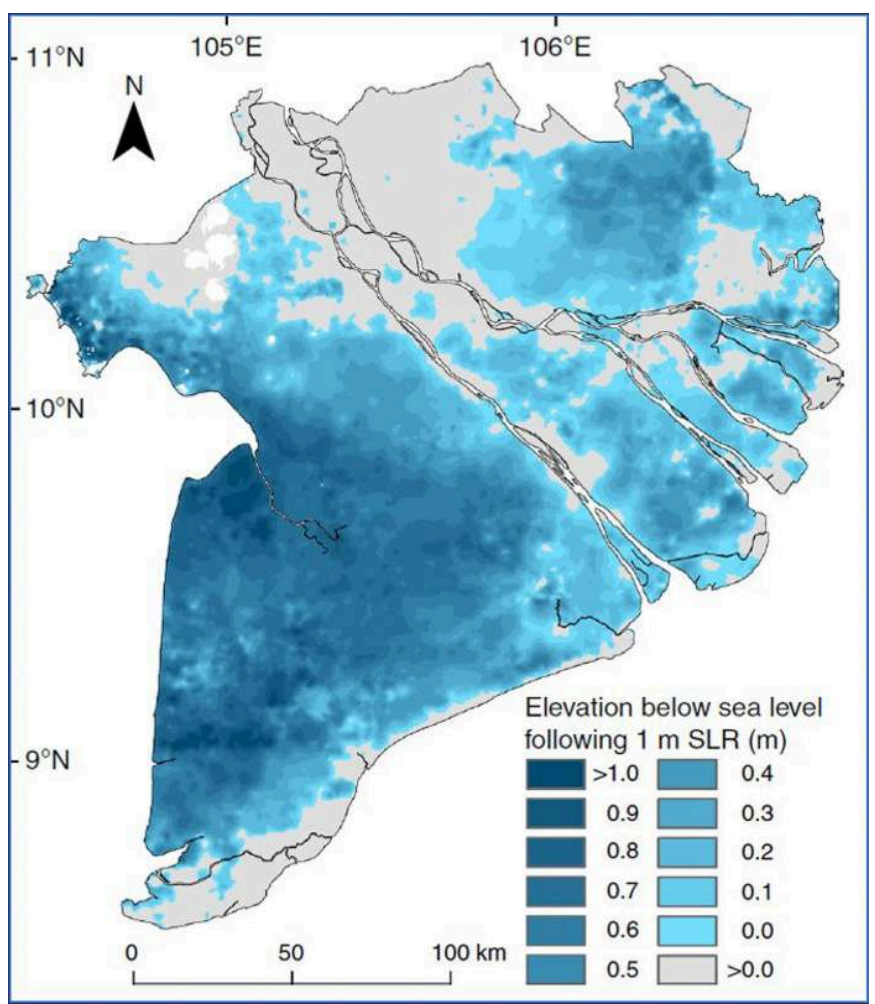

Variation relative : somme de la remontée océanique et de la subsidence. Distribution des zones submergées : en hauteur d'eau.

Source : Minderhoud et al., 2019, p.9.

\section{Quelques pistes?}

d'améliorer les systèmes de drainage urbain par le maintien et le développement des parcs urbains (Vachaud et al., 2018), la création d'une ceinture verte autour de la ville, le long des autoroutes et le long des rivières Saigon et Dong Nai, et probablement par la remise en fonction de navigation fluviale et de drainage des canaux existants. Des décrets ont été pris pour interdire les constructions sur certaines zones humides, mais l'urbanisation en cours de la zone de ThuThiem (illustration 3) et la destruction consécutive de ce grand espace naturel semble ne pas faire de cette interdiction une priorité, même si le projet architectural initial reposait sur une approche d'éco urbanisme.

Au niveau du bâti, il est clair que la préférence devrait être maintenant donnée à la construction de bâtiments en hauteur (pas de niveau habitable sur rue, Duy et al., 2018), à une forte limitation de densité, et au développement de toits et murs végétalisés 
pouvant à la fois réduire l'impact des précipitations intenses en stockant provisoirement l'eau de pluie et limiter l'effet d'ilot de chaleur par l'évapotranspiration des plantes (Rakhshanderhroo et al., 2015).

Au niveau de la protection contre les remontées marines, on peut citer deux projets en cours de réalisation (pour le premier) et d'étude (pour le second).

- Une écluse à marée d'un coût de 450 millions USD sur la rivière Saigon pour éviter les inondations dans le centre-ville. La construction a dû être arrêtée en avril 2018 pour utilisation d'acier de mauvaise qualité.

- Une digue annulaire, d'une hauteur de 2,3 m, qui pourrait encercler la partie intérieure de HCMV (plan MARD Variant en cours d'évaluation). Une étude socioéconomique très intéressante a récemment été publiée (Scussolini et al., 2017) concernant le rapport coûts/avantages du projet. Si en effet le projet voyait jour, la digue protégerait le centre-ville, mais au détriment des zones d'urbanisation des nouveaux quartiers dès lors plus vulnérables.

En plus des mesures techniques, un point essentiel soulevé initialement par Tu et Nitivattananon (2011) puis repris par TranNgoc et al. (2016) est la nécessité d'élaborer un plan d'information d'alerte, basé notamment sur le développement de systèmes de prévisions météorologiques à l'échelle locale, afin d'éliminer au mieux les problèmes de congestion de trafic et d'assurer un moyen préventif de protection des biens. Au plus long terme, et si le risque de submersion se précise, une issue envisagée pourrait être un nouveau plan de développement, avec déplacement des centres vitaux, dans les zones périphériques de plateau, en particulier vers le district de CuChi (Q.T. Nguyen, 2016).

En conclusion le risque de submersion est sérieux pour cette méga cité. Il n'est pas à exclure que d'ici quelques décennies, Ho Chi Minh Ville devienne une île, se limitant à ses quartiers "hauts" (les districts 4, 3, 1 et les quartiers Nord, tous à une altitude supérieure à 1,5 m) à l'intérieur d'une zone marine recouvrant l'essentiel de la LECZ du Bas Mékong. Cette perspective ne relève malheureusement pas de la science-fiction, et nécessite la mise en place rapide d'un groupe de scientifiques, d'économistes et de politiques chargé de l'élaboration d'un plan d'action pour définir les meilleures stratégies entre adaptation et relocalisation en prenant compte des options avancées par Storch et al., (2009).

\section{BIBLIOGRAPHIE}

Abidin H.Z., Gumilar I., Andreas H., Fukuda Y., 2011. Land subsidence of Jakarta and its relation with urban environment. Natural Hazards [En ligne], vol. 59. DOI: https://doi.org/10.1007/ s11069-011-9866-9

Chapman A., Van Pham Dang T., 2018. Climate change is triggering a migrant crisis in Vietnam. The Conversation [En ligne]. 9/11/2018. URL: https://theconversation.com/climate-change-istriggering-a-migrant-crisis-in-vietnam-88791 
Chatel C., Moriconi-Ebrard F., 2018. Les 32 plus grandes agglomérations du monde. Comment l'urbanisation repousse-t-elle ses limites? Confins - Revue franco-brésilienne de géographie [En ligne], vol. 37. DOI: https://doi.org/10.4000/confins.15522

Crutzen P. J., 2004. New directions: The growing urban heat and pollution "island" effect - impact on chemistry and climate. Atmospheric Environment, vol. 38, $\mathrm{n}^{\circ}$ 21, p. 3539-3540.

Dao N. K. and Hoang T. T., 2016. Analysis of changes in precipitation and extremes events in Ho Chi Minh City, Viet Nam. Proceedia Engineering, vol. 142, p. 229-235.

Duy P. N., Lee C., Miles T., Phan N. L. and Le V. T., 2018. Increasing vulnerability to floods in new development areas: evidence from Ho Chi Minh City. International Journal of Climate Change Strategies and Management [En ligne], vol. 10, nº 1, p. 197-212. DOI: https://doi.org/10.1108/ IJCCSM-12- 2016-0169

Ho L. P., Nguyen T., Chau N. X. Q. and Nguyen, K. D., 2014. Integrated urban flood risk management approach in context of uncertainties: Case study Ho Chi Minh City. La Houille Blanche [En ligne], vol. 6, p. 26-33. DOI: https://doi.org/10.1051/lhb/2014059

IPCC, 2015. Climate Change 2014: Synthesis Report. Contribution of Working Groups I, II and III to the Fifth Assessment Report of the Intergovernmental Panel on Climate Change. Core Writing Team, R.K. Pachauri, L.A. Meyer (ed.), Geneva, Switzerland.

Jevrejeva S., Jackon L.P., Riva R.E. M., Grinsted A., Moore J.C., 2016. Coastal sea level rise with warming above $2^{\circ}$ C. PNAS [En ligne], vol 113, n 47, p. 13342-13347 DOI: https://doi.org/10.1073/ pnas. 1605312113

Kontgis C., Schneider A., Fox J., Saksena S., Spencer J. H., Castrence, M., 2014. Monitoring periurbanization in the greater Ho Chi Minh City metropolitan area. Applied Geography, vol. 53, p. 377-388.

McSweeney C., New M. and Lizcano G., 2009. UNDP Climate Change Country Program:Vietnam [Report available online through the Environmental Change Institute, Oxford]. URL: https:// www.geog.ox.ac.uk/research/climate/projects/undpcp/UNDP_reports/Vietnam/ Vietnam.lowres.report.pdf

Minderhoud P. S. J., Coumou L., Erkens G., Middlekoop H., Stouthamer E., 2019. Mekong delta much lower than previously assumed in sea-level rise impact assesments. Nature Communication [En ligne], vol. 10. DOI: https://doi.org/10.1038/s41467-019-11602-1

Minderhoud P.S.J., Erkens G., Pham V.H., Bui V.T., Erban I., Kooi H., Stouthamer E., 2017. Impact of 25 years of groundwater extraction on subsidence in the Mekong Delta. Environ. Res. Letter [En ligne], vol. 12, nº 6. DOI : https://doi.org/10.1088/1748-9326/aa7146

Nguyen Q.T., 2016. The main causes of land subsidence in Ho Chi Minh City. Proceedia Engineering, vol. 142, p. 334-341.

Pham T. T. H., Furukawa M., 2007. Impact of sea level rise on coastal zone of Vietnam. Bulletin of the College of Science, Univ. of the Ryukyus, vol. 84, p. 45-59.

Rakhshanderhroo R. A et al., 2015. Mitigating Urban Heat Island through Green Roofs, Current World Envirt [En ligne], vol. 10, Special Issue. DOI: http://dx.doi.org/10.12944/CWE.10.SpecialIssue1.111

Scussolini P., Tran T. V. T., Koks E., Diaz-Loaiza A., Ho L. P., Lasage R., 2017. Adaptation to sea level rise: a multidisciplinary analysis for Ho Chi Minh City, Vietnam. Water Resources Research [En ligne], vol. 53, n 12, p. 10841-10857. DOI: https://doi.org/10.1002/2017WR021344 
Storch H., N.K. Downes, H.P. Thamm, 2009. Adaptation framework to climate change for the urban area of HoChiMinh City, Conference Paper, Fifth Urban Research Symposium, International Institute for Sustainable Development - IISD-Marseille. http://www.urs2009.net/index.html

Tran Ngoc T. D., Perset M., Strady E., et al., 2016. Ho Chi Minh City growing with water-related challenges Water, Megacities and Global Change: Portraits of 15 Emblematic Cities of the World. Paris, UNESCO - ARCEAU, $27 \mathrm{p}$.

Vachaud G., Quertamp F., Phan T. S. H., et al., 2018. Flood-related risks in Ho Chi Minh City and ways of mitigation. Journal of Hydrology [En ligne], vol. 573, p. 1021-1027. DOI: https://doi.org/ 10.1016/j.jhydrol.2018.02.044

VCAPS, 2013. Climate adaptation strategy for Ho Chi Minh City. Report Vietnam Climate Adaptation PartnerShip Consortium VCAPS [En ligne]. URL: http://www.vcaps.org/assets/uploads/files/ HCMC_ClimateAdaptationStrategy_webversie.pdf

\section{NOTES}

1. Shangai, Ghanzhou (Chine) ; Tokyo (Japon); Jakarta (Indonésie) ; Manille (Philippine) ; Delhi (Inde) ; Ho-Chi-Minh City (Vietnam) ; Séoul (Corée). Chatel \& Moriconi-Ebrard, 2018.

\section{RÉSUMÉS}

La plus grande partie des méga cités du Sud Est Asiatique situées en bordure littorale sont sujettes d'une part à une très forte croissance démographique, d'autre part à un accroissement des risques d'inondations résultant d'une conjonction entre développement urbain mal maitrisé, augmentation de l'intensité des pluies de mousson, élévation du niveau océanique et affaissement du niveau du sol (subsidence). Ho Chi Minh Ville est un exemple typique de cette situation qui peut conduire à moyen-court terme à la submersion de la majorité des quartiers récents construits sur d'anciennes zones de marais ou de rizière. Il est donc essentiel de mettre en œuvre des stratégies de gestion des risques adaptées et de développer le territoire de manière durable

\section{INDEX}

Mots-clés : Vietnam, méga cité, inondation, urbanisation, changement climatique, subsidence Thèmes : Sur le Champ - Sur le Terrain

\section{AUTEURS}

\section{GEORGES VACHAUD}

Georges Vachaud est directeur de recherche Emérite au CNRS et membre de l'UMR IGE. Il a récemment publié : 
- Vachaud G., Quertamp F., Phan T. S. H., et al., 2018. Flood-related risks in Ho Chi Minh City and ways of mitigation. Journal of Hydrology [En ligne], vol. 573, p. 1021-1027. DOI: https://doi.org/ 10.1016/j.jhydrol.2018.02.044

- Tran Ngoc T. D., Perset M., Strady E., et al., 2016. Ho Chi Minh City growing with water-related challenges Water, Megacities and Global Change: Portraits of 15 Emblematic Cities of the World. Paris, UNESCO - ARCEAU, $27 \mathrm{p}$.

- Chang F.J., Tsai Y.H, ChenP.A., et al., 2015. Modeling water quality in an urban river using hydrological factors - Data driven approaches. Journal of Environmental Management [En ligne], vol. 151, p. 87-96. DOI: https://doi.org/10.1016/j.jenvman.2014.12.014

\section{NICOLAS GRATIOT}

Nicolas Gratiot directeur de recherche à l'IRD et membre de l'UMR IGE. Il a récemment publié : - Le H.A., Gratiot N., Santini W., et al., 2020. Suspended sediment properties in the Lower Mekong River (LMR) from fluvial to estuarine environments. Estuarine Coastal and Shelf Science, vol. 233. - Vachaud G., Quertamp F., Phan T. S. H., et al., 2018. Flood-related risks in Ho Chi Minh City and ways of mitigation. Journal of Hydrology [En ligne], vol. 151, p. 87-96. DOI: https://doi.org/10.1016/ j.jenvman.2014.12.014

- Besset M., Gratiot N., Anthony E.J., et al., 2019. Mangroves and shoreline erosion in the Mekong River delta, Viet Nam. Estuarine, Coastal and Shelf Science [En ligne], vol. 226. DOI: https://doi.org/ 10.1016/j.ecss.2019.106263

\section{TIEN DUNG TRAN NGOC}

Tien Dung Tran Ngoc est chercheur associé à l'Asian Research Center on Water (CARE-Rescif), Ho Chi Minh City University of Technology. Il a récemment publié:

- Tran Ngoc T. D., Trang N. G., Ruin I., Gratiot N., 2018. Ngập ở TP. HCM: Người dân lo gì nhất? (Flooding in HCM City: what do the residents worry about?). Tuoi Tre Weekly Magazine [En ligne\}. URL: https://cuoituan.tuoitre.vn/tin/van-de-su-kien/20181017/ngap-o-tphcm-nguoi-dan-lo-ginhat/1463117.html (in Vietnamese)

- Vachaud G., Quertamp F., Phan T. S. H., et al., 2018. Flood-related risks in Ho Chi Minh City and ways of mitigation. Journal of Hydrology [En ligne], vol. 151, p. 87-96. DOI: https://doi.org/10.1016/ j.jenvman.2014.12.014

- Tran Ngoc T. D., Perset M., Strady E., et al., 2016. Ho Chi Minh City growing with water-related challenges Water, Megacities and Global Change: Portraits of 15 Emblematic Cities of the World. Paris, UNESCO - ARCEAU, 27 p. 